\title{
Effects of tranilast on the epithelial-to-mesenchymal transition in peritoneal mesothelial cells
}

\author{
Seok Hui Kang ${ }^{1(i)}$, Sang Woon $\mathrm{Kim}^{2}{ }^{(\mathbb{D}}$, Keuk Jun $\mathrm{Kim}^{3(\mathbb{D}}$, Kyu Hyang Cho ${ }^{1(i)}$, Jong Won Park ${ }^{1(\mathbb{D})}$, \\ Chan-Duck Kim ${ }^{4, *}$, Jun Young $\mathrm{Do}^{1, *(\mathbb{D})}$ \\ 'Division of Nephrology, Department of Internal Medicine, Yeungnam University Medical Center, Daegu, Republic of Korea \\ ${ }^{2}$ Division of Gastro-Enterology, Department of Surgery, Yeungnam University Hospital, Daegu, Republic of Korea \\ ${ }^{3}$ Department of Biomedical Laboratory Science, Daekyeung University, Gyeongsan, Republic of Korea \\ ${ }^{4}$ Division of Nephrology, Department of Internal Medicine, Kyungpook National University Hospital, School of Medicine, Kyungpook \\ National University, Daegu, Republic of Korea
}

Background: We investigated the effects of tranilast on epithelial-to-mesenchymal transition (EMT) in an animal model and on the EMT signaling pathway in human peritoneal mesothelial cells (HPMCs).

Methods: We performed in vitro studies (cytotoxicity, cell morphology, and western blot analyses) on HPMCs from human omenta, along with in vivo studies (peritoneal membrane function and morphometric and immunohistochemical analyses) on Sprague Dawley rats. Thirty-two rats were divided into three groups: control (C) group (peritoneal dialysis [PD] catheter but not infused with dialysate), PD group (4.25\% glucose-containing dialysate), and $\mathrm{PD}+$ tranilast group (4.25\% glucose-containing dialysate along with tranilast).

Results: In in vitro experiments, transforming growth factor-beta 1 (TGF- $\beta 1$ ) increased $\alpha$-smooth muscle actin and Snail expression and reduced E-cadherin expression in HPMCs. TGF- $\beta 1$ also reduced cell contact, induced a fibroblastoid morphology, and increased phosphorylation of Akt, Smad2, and Smad3 in HPMCs. Tranilast significantly inhibited TGF- $\beta 1$-induced EMT and attenuated these morphological changes in HPMCs. In in vivo studies, after 6 weeks of experimental PD, the peritoneal membrane was significantly thicker in the PD group than in the $C$ group. Tranilast protected against PD-induced glucose mass transfer change and histopathological changes in rats.

Conclusion: Tranilast prevented EMT both in HPMCs triggered with TGF- $\beta 1$ and in rats with PD-induced peritoneal fibrosis. Thus, tranilast may be considered a therapeutic intervention that enables long-term PD by regulating TGF- $\beta 1$ signaling pathways.

Keywords: Epithelial-mesenchymal transition, Fibrosis, Peritoneal dialysis, Peritoneum, Tranilast

Received April 30, 2019; Revised July 3, 2019; Accepted July 9, 2019

Edited by Won Kim, Chonbuk National University, Jeonju, Republic of Korea

\section{Correspondence:}

Jun Young Do

Department of Internal Medicine, Yeungnam University Medical Center, 170 Hyeonchung-ro, Nam-gu, Daegu 42415, Republic of Korea.E-mail:jydo@med.yu.ac.kr

Chan-Duck Kim

Department of Internal Medicine, Kyungpook National University Hospital, School of Medicine, Kyungpook National University, 130 Dongdeok-ro, Jung-gu, Daegu 41944, Republic of Korea. E-mail: drcdkim@knu.ac.kr

*Chan-Duck Kim and Jun Young Do contributed equally to this study.

Copyright @ 2019 by The Korean Society of Nephrology

(a) This is an open-access article distributed under the terms of the Creative Commons Attribution Non-Commercial License (http://creativecommons.org/ licenses/by-nc-nd/4.0/), which permits unrestricted non-commercial use, distribution, and reproduction in any medium, provided the original work is properly cited. 


\section{Introduction}

According to the United States Renal Data System (USRDS), chronic kidney disease affects $12 \%$ of the general population [1]. The proportion of new end-stage renal disease patients who require renal replacement therapy has reached 357 per million. Peritoneal dialysis (PD) is an established modality among renal replacement therapies. In the United States, 185,000 patients have received PD [1].

Ultrafiltration failure and/or fibrosis of peritoneal membranes are the most significant hurdles to successful long-term PD [2]. Transforming growth factor-beta 1 (TGF- $\beta 1$ )-induced epithelial-to-mesenchymal transition (EMT) plays a key role in peritoneal membrane changes [3]. During EMT, peritoneal mesothelial cells lose their epithelial phenotype and acquire new mesenchymal characteristics. Because EMT is associated with excessive production of fibrous connective tissue, effectively attenuating EMT is important for maintaining PD. Icodextrin and bicarbonate/low-glucose degradation product dialysates have been introduced to overcome EMT; however, their effectiveness in suppressing EMT remains controversial [4-7].

Tranilast is a well-known anti-allergic drug used clinically for bronchial asthma, allergic rhinitis, hypertrophic scarring, and scleroderma [8-10]. Moreover, many studies have demonstrated that tranilast has anti-proliferative, anti-cancer, anti-oxidant, cardiovascular-protective, and immune-modulating effects [11], suggesting that it may be effective for treatment of various pathological conditions. Tranilast has also been demonstrated to suppress TGF- $\beta 1$-induced EMT in renal tubular epithelial cells and tumor cells [12-14]. Similarly, Saito et al [15] recently reported that tranilast inhibited TGF- $\beta 1$-induced fibrosis in human peritoneal mesothelial cells (HPMCs). Although these data suggest that tranilast may prevent EMT in peritoneal mesothelial cells, few studies have demonstrated these effects in an animal model [16].

Here, we evaluated the effects of tranilast on the EMT signaling pathway in both HPMCs and an animal model.

\section{Methods}

In vitro study

\section{HPMC culture and treatment conditions}

HPMCs were isolated as described previously [17]. Tissue collection was approved by the Institutional Review Board of Yeungnam University Medical Center (approval number: 2019-04-047). Informed consent was obtained from each participant. In vitro experiments were performed on cells after 1 to 2 passages. HPMCs were incubated with serum-free M199 medium for 1 day. Subsequently, the medium was substituted with M199 medium supplemented with $20 \%$ fetal bovine serum containing TGF- $\beta 1$ (2.0 ng/mL, \#240-B; R\&D Systems, Minneapolis, MN, USA) with or without tranilast (100 $\mu \mathrm{M}$; JW Pharm., Co., Seoul, Korea) for 1 or 7 days. In preliminary experiments, tranilast exhibited its maximum effect on TGF- $\beta 1$ induced EMT at a concentration of $100 \mu \mathrm{M}$ (Supplementary Fig. 1; available online).

\section{Cytotoxicity assay}

The cytotoxicity of tranilast was evaluated with the Cell Counting Kit-8 (CCK-8) (Abcam, Cambridge, MA, USA). HPMCs (10,000 cells) were grown in a 96-well plate for 24 hours and then were treated with tranilast $(0,25,50,100$, 200 , or $400 \mu \mathrm{M}$ ) for another 24 hours. The CCK-8 solution was added to each well, and optical density at 450 nm (OD450) was measured between 1 and 4 hours. The results of the cytotoxicity assay are shown in Supplementary Fig. 2.

\section{Cell morphology and Western blotting}

Cell morphology was analyzed with a phase-contrast microscope (Nikon DIAPHOT 300; Nikon, Tokyo, Japan), and images were captured with a digital camera (AxioCam HRC; Carl Zeiss, Göttingen, Germany).

Immunoblotting was performed as described previously [17]. Briefly, HPMCs were washed with cold phosphate-buffered saline, exposed to trypsin, and pelleted by centrifugation at $700 \mathrm{~g}$ at $4^{\circ} \mathrm{C}$. The pellets were resuspended in lysis buffer. The preparation was then clarified by centrifugation, and the supernatant was saved as the whole-cell lysate. The protein samples were mixed in sodium dodecyl sulfate (SDS) reducing buffer, boiled, electrophoresed through a $10 \%$ reducing SDS-polyacrylamide 
gel, and electroblotted in $20 \%$ methanol, $25 \mathrm{mM}$ Tris, and $192 \mathrm{mM}$ glycine onto a nitrocellulose membrane. The membranes were blocked in $5 \%(\mathrm{w} / \mathrm{v})$ nonfat milk powder in Tris-buffered saline for 1 hour at room temperature.

The blots were incubated overnight with primary antibody at $4^{\circ} \mathrm{C}$. The primary antibodies were $\beta$-actin (1:2,000 dilution, A1978; Sigma-Aldrich, St. Louis, MO, USA), mouse monoclonal anti- $\alpha$-smooth muscle actin ( $\alpha$-SMA, 1:1,000 dilution, A5228; Sigma-Aldrich), mouse monoclonal anti-E-cadherin (1:1,000 dilution, 610181; BD Biosciences, Lexington, KY, USA), and the following antibodies from Cell Signaling Technology (Danvers, MA, USA), all at 1:1,000 dilution: rabbit monoclonal antiglyceraldehyde 3-phosphate dehydrogenase (GAPDH, \#2118), mouse monoclonal anti-Snail (\#3895), rabbit monoclonal anti-phosphorylated-Smad2 (p-Smad2, \#3108), mouse monoclonal anti-Smad2 (\#3103), rabbit monoclonal anti-phosphorylated-Smad3 (p-Smad3, \#8769), rabbit polyclonal anti-Smad3 (\#9513), rabbit polyclonal anti-Smad4 (\#9515), rabbit polyclonal anti-Smad6 (\#9519), rabbit monoclonal anti- $\beta$-catenin (\#9582), rabbit monoclonal anti-phosphorylated-Akt (pAkt, \#4060), and rabbit monoclonal anti-Akt (\#4685). The secondary antibodies (goat anti-rabbit immunoglobulin (Ig) G-horseradish-peroxidase [A16096, Invitrogen; Thermo Fisher Scientific Inc., Waltham, MA, USA] and goat anti-mouse IgG-horseradish-peroxidase [sc-516102; Santa Cruz Biotechnology, Santa Cruz, CA, USA], both at 1:2,000 dilution) to each primary antibody were used, and an enhanced chemiluminescence system (Thermo Fisher Scientific Inc.) was used to detect the immunoreactive bands.

In vivo study

\section{Animal experiments}

Thirty-two male Sprague Dawley rats (200-250 g) were obtained from Orient Corporation (Seoul, Korea). All animal procedures were approved by the Institutional Review Board of Yeungnam University College of Medicine (approval number: YUMC-AEC2013-027) and were in accordance with the Guide for the Care and Use of Laboratory Animals. Peritoneal catheters were inserted as previously described [18]. Rats were divided into three treatment groups: 1$)$ the control (C) group $(n=8)$, which underwent catheter implantation without infusions; 2) the PD group $(\mathrm{n}=12)$, which received infusions with a $4.25 \%$ glucose-containing dialysate (Dianeal ${ }^{\circledR}$; Baxter Healthcare, Woodlands, Singapore); and 3) the PD + tranilast group $(\mathrm{n}=12)$, which received infusions with a $4.25 \%$ glucose-containing dialysate and cotreatment with tranilast. For 6 weeks, rats in the PD group and the PD + tranilast group received a total of $25 \mathrm{~mL}$ of $4.25 \%$ glucose-containing dialysate (twice daily). The infusions contained cefazolin $(1 \mathrm{~g} / \mathrm{L})$ and amikacin $(100 \mathrm{mg} / \mathrm{L})$ as antibiotics. The tranilast-treated rats were fed a chow diet containing tranilast at a concentration of $0.8 \%(w / w)$.

\section{Peritoneal membrane function test}

Peritoneal membrane function was evaluated by a 4-hour peritoneal equilibrium test (PET) with $4.25 \%$ glucose-containing dialysate at 0 day and 6 week. After 4 hours, a sample of peritoneal dialysate was obtained by aspiration, and a sample of blood was obtained by heart puncture. The glucose mass transfer from the peritoneal cavity to the blood was assessed as reported previously [16].

Morphometric and immunohistochemical analyses of the peritoneum

At the end of 6 week, the rats were killed, and the parietal peritoneum of the abdominal wall was sampled for morphometric and histological analyses. The parietal peritoneum was assessed by hematoxylin and eosin (H\&E) staining as previously described [17]. Fibrosis was visualized with Masson's trichrome stain. Immunohistochemical staining experiments for $\alpha$-SMA, fibronectin, and TGF- $\beta 1$ were performed with rabbit polyclonal anti- $\alpha$-SMA (ab5694; Abcam), rabbit monoclonal antifibronectin (ab199056; Abcam), and rabbit monoclonal anti-TGF- $\beta 1$ [EPR21143] (ab215715; Abcam) primary antibodies, respectively.

\section{Statistical analysis}

Two rats in the $\mathrm{C}$ group that died during baseline PET were excluded from the analysis. IBM SPSS Statistics ver. 19.0 was used to analyze the data (IBM Corp., Armonk, NY, USA). Data are expressed as the mean and standard error of the mean. Groups were compared by the Kruskal-Wallis or Mann-Whitney rank-sum test. Differences 


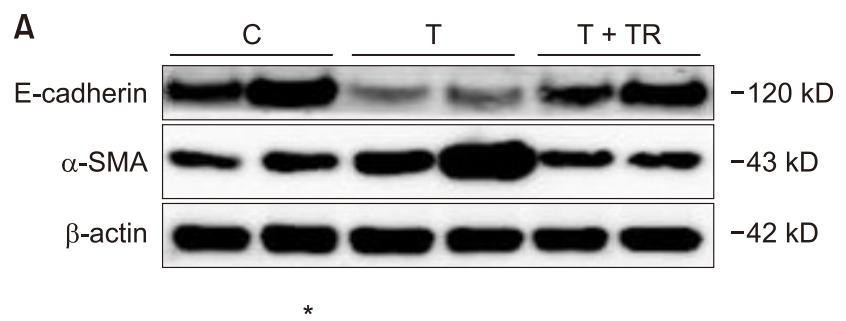

B
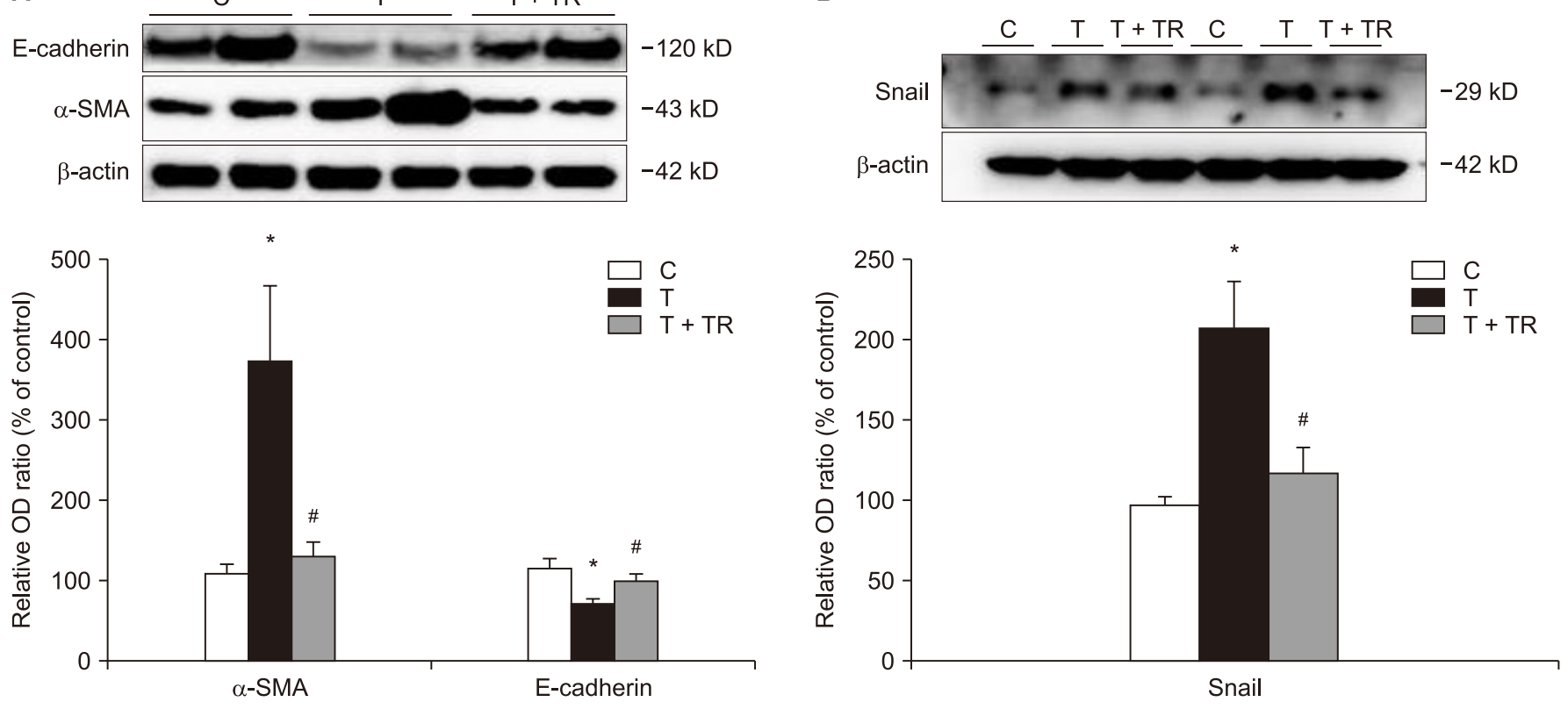

Figure 1. Western blot analysis of epithelial-to-mesenchymal transition markers in human peritoneal mesothelial cells (HPMCs). HPMCs were treated with transforming growth factor-beta 1 (TGF- $\beta 1$ ) in the presence or absence of tranilast (100 $\mu \mathrm{M})$ for 24 hours. Quantification is relative to the control and normalized to $\beta$-actin expression. TGF- $\beta 1$ increased the protein expression of $\alpha$-smooth muscle actin $(\alpha-S M A)(A)$ and Snail $(B)$ and reduced the protein expression of E-cadherin (A) in HPMCs. Tranilast reversed the changes in these markers. $n$ $=4$ per group.

C, control; OD, optical density; T, HPMCs with TGF- $\beta 1$ treatment; T + TR, HPMCs with TGF- $\beta 1$ and tranilast cotreatment.

${ }^{*} P<0.05$ compared to the $\mathrm{C}$ group, ${ }^{\#} P<0.05$ compared to the T group.

between the two time points (initiation and six weeks) were compared by the Wilcoxon singed-rank test. A $P$ value $<0.05$ was considered significant.

\section{Results}

\section{Tranilast attenuates TGF- $\beta 1$-induced EMT in vitro}

We evaluated the effects of tranilast on TGF- $\beta 1$-induced EMT in vitro. As shown in Fig. 1, exposure of HPMCs to TGF- $\beta 1$ downregulated the epithelial cell marker E-cadherin but upregulated the mesenchymal marker $\alpha$-SMA and the EMT-inducing transcription factor Snail. Tranilast cotreatment significantly reversed each of these TGF$\beta 1$-induced changes.

\section{Tranilast attenuates TGF- $\beta 1$-induced morphological changes and signaling}

The morphological changes in HPMCs from day 1 to 7 are shown in Fig. 2. TGF- $\beta 1$ triggered cell contact losses and fibroblastoid morphological changes. These mor- phological changes were evident after 2 days, with greater deterioration during the subsequent days. On day 2 , tranilast was administered at a dose of 1 to $100 \mu \mathrm{M}$, which attenuated the morphologic changes. A high dose of tranilast exhibited a sustained protective effect from day 2 to 7.

\section{Tranilast attenuates TGF- $\beta 1$-induced Smad, Akt, and} $\beta$-catenin signaling

Treatment of HPMCs with TGF- $\beta 1$ (24 hours) significantly increased the phosphorylation of Smad2 and Smad3 (Fig. 3). However, after cotreatment with tranilast, a significant decrease in TGF- $\beta 1$-induced Smad 2 and Smad3 phosphorylation was observed. Smad4 expression increased and Smad6 expression decreased in cells treated with TGF- $\beta 1$, but tranilast reversed these effects.

Fig. 4 shows a significant increase in phosphorylation of Akt in the presence of TGF- $\beta 1$. TGF- $\beta 1$ also increased the expression of $\beta$-catenin, an EMT-promoting transcriptional factor. Tranilast reduced these changes. 


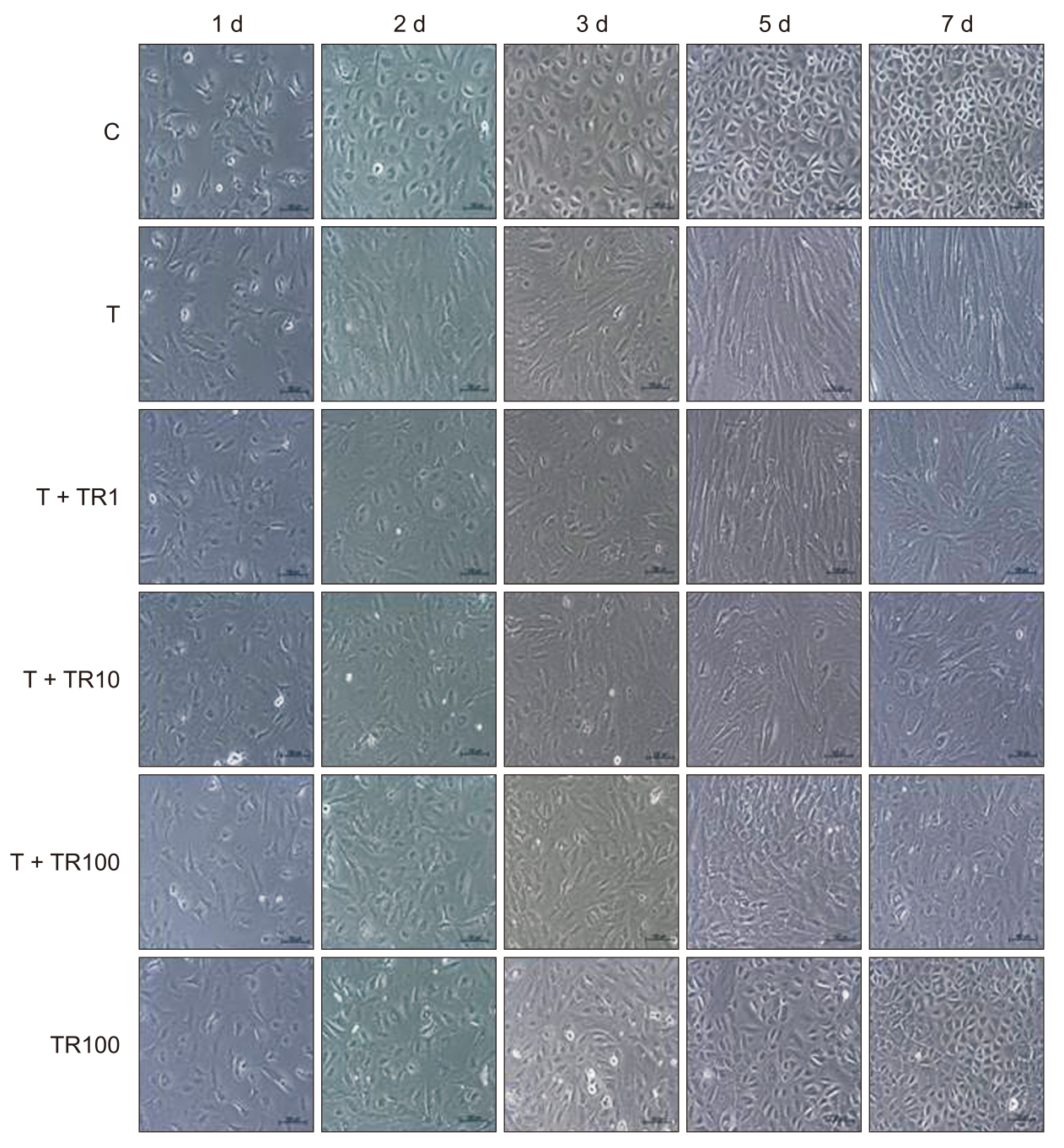

Figure 2. Effects of tranilast on transforming growth factor-beta 1 (TGF- $\beta 1$ induced morphological changes.

The effects of tranilast on TGF- $\beta 1$-induced changes in human peritoneal mesothelial cell (HPMC) morphology were observed by phase-contrast microscopy. HPMCs treated with control buffer or tranilast exhibited a cobblestone appearance (C group), while those exposed to TGF- $\beta 1$ exhibited a spindle shape (T group). HPMCs exposed to TGF- $\beta 1$ and tranilast reverted to a cobblestone appearance ( $T+T R 1$, $T$ + TR10, and T + TR100 groups).

C, control; OD, optical density; T, HPMCs exposed to TGF- $\beta 1$; T + TR1, HPMCs exposed to TGF- $\beta 1$ and tranilast $1 \mu \mathrm{M}$; T + TR10, HPMCs exposed to TGF- $\beta 1$ and tranilast $10 \mu \mathrm{M} ; \mathrm{T}+\mathrm{TR} 100$, HPMCs exposed to TGF- $\beta 1$ and tranilast $100 \mu \mathrm{M}$; TR100, HPMCs exposed to tranilast $100 \mu \mathrm{M}$.
Tranilast protects against PD-induced glucose mass transfer change in vivo model

The body weights of the animals increased during the study; however, no significant differences were detected among the three groups (Table 1). There was no significant difference among the groups in glucose mass transfer at initiation of PD (day 0). However, after 6 weeks, the glucose mass transfer level was significantly higher than the baseline value ( 0 day) in the PD group but not in the $\mathrm{C}$ or PD + tranilast group (Table 1). These results revealed that tranilast attenuated EMT-related changes not only at the molecular level, but also at the functional level.

\section{Tranilast attenuates PD-induced histopathological changes in vivo}

After 6 weeks of experimental PD, the peritoneal mem- brane was significantly thicker in the PD group than in the $\mathrm{C}$ group, but this change was prevented by tranilast. In addition, deposition of collagen was greater in the PD group than in the $\mathrm{C}$ group, but cotreatment with tranilast suppressed this effect (Fig. 5).

We also monitored the protein levels of the epithelial cell marker E-cadherin and the mesenchymal marker $\alpha$-SMA in the peritoneal membrane (Fig. 6). Similar to the in vitro results, E-cadherin expression was lower and $\alpha$-SMA expression was higher in the PD group than in the $\mathrm{C}$ group. Tranilast was able to reverse these changes.

To investigate the fibrotic response in the peritoneum after PD, we used immunohistochemistry to evaluate the expression of $\alpha$-SMA, fibronectin, and TGF- $\beta 1$ in parietal peritoneal tissues. The protein levels of $\alpha$-SMA and fibronectin (fibrotic components) and TGF- $\beta 1$ (a key signal of EMT and fibrosis) were greater in the PD group than in the $\mathrm{C}$ group. Treatment with tranilast reduced the chang- 

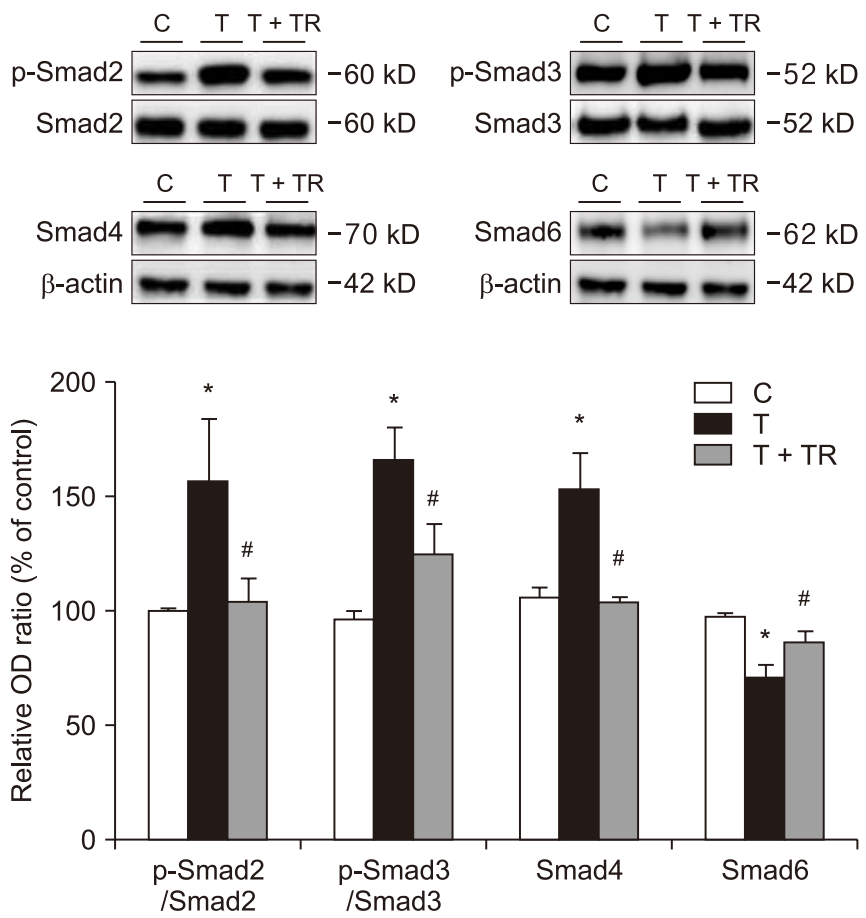

Figure 3. Effects of tranilast on the Smad-dependent signaling pathway. Western blot analysis was performed on human peritoneal mesothelial cells (HPMCs) exposed to transforming growth factorbeta 1 (TGF- $\beta 1$ ) in the presence or absence of tranilast $(100 \mu \mathrm{M})$ for 24 hours. Quantification is relative to the control and normalized to $\beta$-actin expression. TGF- $\beta 1$ increased the $p$-Smad2/Smad2 and $p$ Smad3/Smad3 protein expression ratios, increased Smad4 protein expression, and reduced Smad6 protein expression. Tranilast reversed these changes. $n=4$ per group.

C, control; OD, optical density; T, HPMCs with TGF- $\beta 1$ treatment; T + TR, HPMCs with TGF- $\beta 1$ and tranilast cotreatment.

${ }^{\star} P<0.05$ compared to the $C$ group, ${ }^{\#} P<0.05$ compared to the $T$ group.

es in these markers. These findings extended the in vitro results by revealing that tranilast could also attenuate the TGF- $\beta 1$ signaling pathway when it was induced by PD in vivo (Supplementary Fig. 3).

\section{Discussion}

Our results indicated that tranilast inhibits signaling pathways involving Smad and Akt/ $\beta$-catenin, thereby suppressing the EMT in peritoneal mesothelial cells. In vivo, tranilast reduced the thickness of the peritoneal membrane and the expression of mesenchymal markers but increased the expression of an epithelial cell marker.

Glucose degradation products and/or advanced glycation end-products in dialysates can upregulate TGF- $\beta 1$,
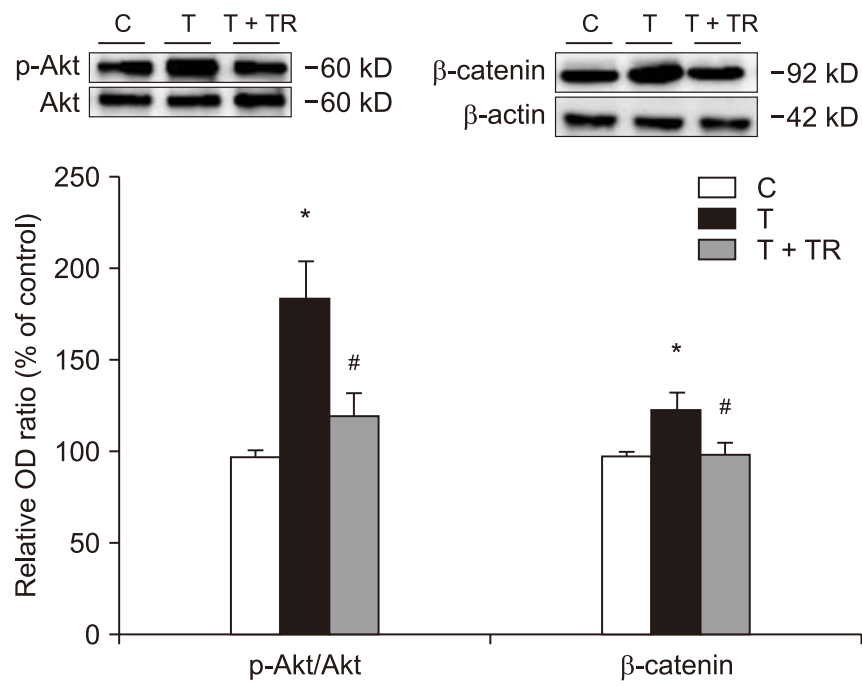

Figure 4. Effects of tranilast on the Akt/ $\beta$-catenin signaling pathway. Western blot analysis was performed on human peritoneal mesothelial cells (HPMCs) exposed to transforming growth factorbeta 1 (TGF- $\beta 1$ ) in the presence or absence of tranilast $(100 \mu \mathrm{M})$ for 24 hours. Quantification is relative to the control and normalized to $\beta$-actin expression. TGF- $\beta 1$ increased phosphorylated-Akt (p-Akt)/Akt protein expression ratio and $\beta$-catenin protein expression. Tranilast reversed these changes. $n=4$ per group.

C, control; OD, optical density; T, HPMCs with TGF- $\beta 1$ treatment; T + TR, HPMCs with TGF- $\beta 1$ and tranilast cotreatment.

${ }^{\star} P<0.05$ compared to the $C$ group, ${ }^{\#} P<0.05$ compared to the $T$ group.

Table 1. Results of the peritoneal equilibrium test

\begin{tabular}{lcc}
\hline \multicolumn{1}{c}{ Variable } & \multicolumn{1}{c}{ Initiation } & 6 weeks of PD \\
\hline Body weight $(g)$ & & \\
C group & $259.8 \pm 5.3$ & $412.4 \pm 13.7^{*}$ \\
PD group & $260.0 \pm 2.2$ & $394.8 \pm 4.7^{*}$ \\
PD + TR group & $253.9 \pm 3.3$ & $399.3 \pm 9.4 *$ \\
Glucose mass transfer/body weight $(\mathrm{mg} / \mathrm{g})$ & \\
C group & $1.71 \pm 0.02$ & $1.71 \pm 0.24$ \\
PD group & $1.77 \pm 0.05$ & $1.94 \pm 0.09 *$ \\
PD + TR group & $1.78 \pm 0.03$ & $1.89 \pm 0.34$ \\
\hline
\end{tabular}

Statistical significance was determined with the Kruskal-Wallis test and Wilcoxon signed-rank test. Data are expressed as mean \pm standard error.

C, control; PD, peritoneal dialysis; PD + TR, peritoneal dialysis group with tranilast cotreatment.

$\star P<0.05$ compared with the baseline value in the same group.

which then induces EMT in peritoneal mesothelial cells $[5,19]$. TGF- $\beta 1$ phosphorylates Smad2 and Smad3, which then bind separately to Smad4 to form a Smad complex [20]. This complex translocates to the nucleus, where it produces factors associated with EMT and regulates 

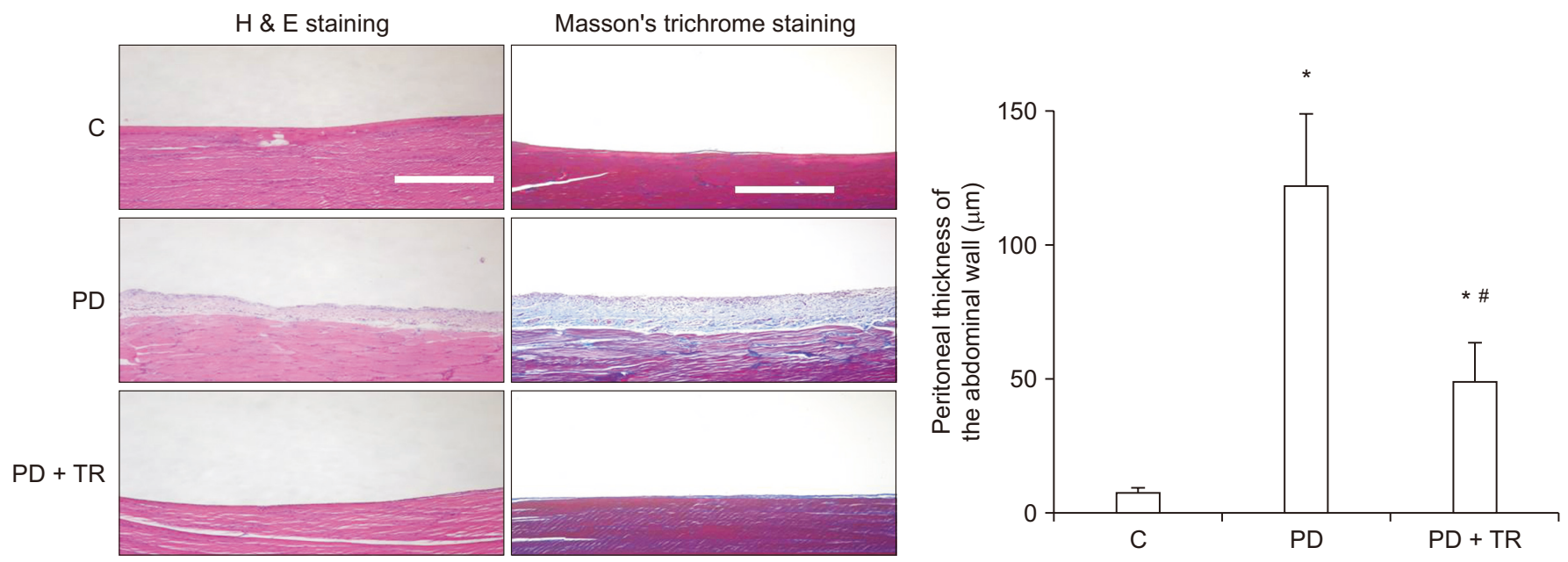

Figure 5. Morphological changes in the peritoneum. Histopathology was assessed 6 weeks after initiation of peritoneal dialysis in rats. Hematoxylin and eosin (H\&E) staining revealed that the peritoneal thickness of the abdominal wall was greater in the peritoneal dialysis (PD) group than in the $\mathrm{C}$ group (magnification 100x; white scale bar, $100 \mu \mathrm{m}$ ) and was lesser in the PD + TR group than in in the PD group. Masson's trichrome staining (magnification 100x; white scale bar, $100 \mu \mathrm{m}$ ) revealed similar results. Data for peritoneal thickness are expressed as mean \pm standard error. The $n$ values for the C, PD, and PD + TR groups were 8, 12, and 12, respectively.

$\mathrm{C}$, control; PD + TR, peritoneal dialysis group with tranilast cotreatment.

$\star P<0.05$ compared to the $C$ group at 6 weeks, ${ }^{\#} P<0.05$ compared to the $P D$ group.
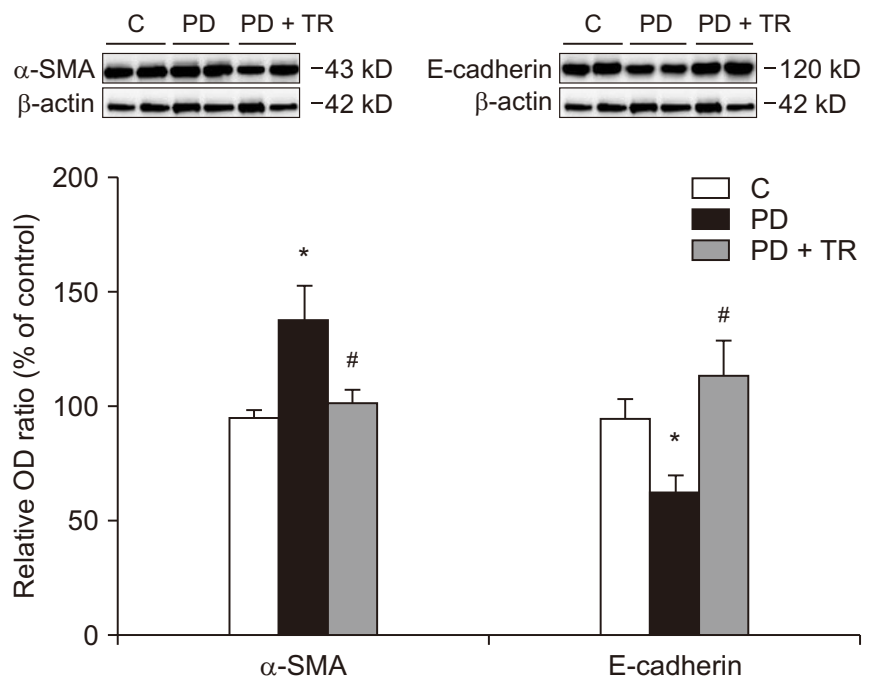

Figure 6. Epithelial-to-mesenchymal transition markers in the parietal peritoneum. Western blot analysis of the parietal peritoneum was performed 6 weeks after initiation of peritoneal dialysis in rats. Quantification is relative to the control and normalized to $\beta$-actin expression. The protein expression of $\alpha$-smooth muscle actin $(\alpha-\mathrm{SMA})$ was greater in the peritoneal dialysis (PD) group than in the $\mathrm{C}$ group, while the protein expression of $\mathrm{E}$-cadherin was lesser in the PD group than in the $\mathrm{C}$ group. These changes were reversed in the PD + TR group. The relative optical density (OD) values are expressed as mean \pm standard error. The $n$ values for the $C$, PD, and PD + TR groups were 8, 12, and 12, respectively.

$\mathrm{C}$, control; $\mathrm{PD}+\mathrm{TR}$, peritoneal dialysis group with tranilast cotreatment. ${ }^{*} P<0.05$ compared to the $C$ group, ${ }^{*} P<0.05$ compared to the $P D$ group.
Smad6, an inhibitory Smad.

Tranilast was initially used to treat allergies and fibrotic disorders such as scleroderma and hypertrophic scarring [8-10]. Kaneko et al [16] reported that tranilast inhibited high-glucose-induced TGF- $\beta 1$ mRNA production both in vitro and in vivo. Tranilast treatment also reduced the levels of fibrogenic factors in samples from three patients. However, these preliminary results did not include an examination of the affected signaling pathways. We demonstrated that tranilast suppressed the phosphorylation of Smad2 and Smad3, reduced Smad4 expression, and increased Smad6 expression. These data indicate that tranilast inhibits the TGF- $\beta 1$-Smad pathway and attenuates EMT in peritoneal mesothelial cells. In addition, tranilast attenuated TGF- $\beta 1$-induced morphologic changes, including loss of cell contacts. These effects were also demonstrated in vivo. PD significantly increased the thickness of the parietal peritoneum and deposition of collagen, but tranilast reduced these changes.

In addition to the Smad-dependent signaling pathway, other signaling cascades are activated by TGF- $\beta 1$, including MAPK, Rho-like GTPase, and PI3K/Akt pathways $[21,22]$. TGF- $\beta 1$ activates PI3K, which induces phosphorylation of Akt. Activated Akt increases the expression of $\beta$-catenin and Snail, which promotes the EMT phenotype 
[23]. In our study, tranilast inhibited not only the TGF- $\beta 1$ induced Smad-dependent pathway, but also the PI3K/ Akt pathway.

There have been many developments in interventions for peritoneal fibrosis and studies of associated mechanisms. Although TGF- $\beta 1$ and/or the renin-angiotensin system are the classical mediators of peritoneal fibrosis, recent reports have revealed the significance of other signaling pathways in peritoneal fibrosis [24-28]. Ji et al [25] investigated the role of $\beta$-catenin, a cytoskeletal protein, in high-glucose dialysate-induced EMT pathology. They found that inhibiting $\beta$-catenin reversed the expression of high-glucose-induced EMT markers. In another study, high-glucose dialysate was reported to induce phosphorylation of glycogen synthase kinase (GSK)-3 $\beta$ and thus inhibit degradation of $\beta$-catenin [26]. Ultimately, this $\beta$-catenin signaling was implicated in EMT. Yan et al [26] investigated the association between high-glucose dialysate treatment and GSK-3 $\beta$ phosphorylation and demonstrated that tamoxifen downregulated the GSK-3 $\beta$ / $\beta$-catenin axis. Kazama et al [27] focused on the function of mast cells in progression of peritoneal fibrosis. They found that chronic kidney disease increased the number of mast cells in the peritoneum of rats, and that tranilast, a mast cell stabilizer, suppressed the progression of peritoneal fibrosis [27].

Recent studies have revealed the significance of microRNA-29 in the pathophysiology of fibrosis [28]. An inverse relationship between microRNA-29 and TGF- $\beta 1$ expression has been demonstrated in a unilateral ureteral obstruction model and other cell types [28,29]. Although there are no data regarding the association between microRNA-29 and tranilast in HPMCs, Chuang and Khorram [30] reported that tranilast induced the expression of microRNA-29, which attenuated fibrotic marker levels in leiomyoma smooth muscle cells. These data reveal that tranilast is a therapeutic option for peritoneal fibrosis through various signaling pathways.

Our PET results demonstrated that 6 weeks of PD significantly increased glucose mass transfer. However, there was no significant difference in glucose mass transfer after 6 weeks in the group treated with tranilast. Thus, in addition to altering the molecular and morphologic responses to TGF- $\beta 1$, tranilast also prevented the functional changes induced by PD.

In conclusion, this study demonstrated that tranilast can prevent EMT in rats with PD-induced peritoneal fibrosis, as well as in HPMCs treated with TGF- $\beta 1$. The mechanism of action of tranilast involved regulating TGF- $\beta 1$ signaling pathways. Thus, tranilast may be a therapeutic intervention to enable long-term PD.

\section{Conflicts of interest}

The authors declare that they have no competing interests.

\section{Acknowledgments}

This work was supported by the Medical Research Center Program (2015R1A5A2009124) through the National Research Foundation of Korea funded by the Ministry of Science, ICT, and Future Planning.

\section{Authors' contributions}

Seok Hui Kang designed/performed the study and wrote the manuscript. Sang Woon Kim provided intellectual content and critically assessed the work. Keuk Jun Kim interpreted the data. Kyu Hyang Cho and Jong Won Park drafted and revised the article. Jun Young Do analyzed and interpreted data and drafted and revised the article. Chan-Duck Kim revised the article and approved the final version to be published.

\section{References}

[1] U.S. Renal Data System. USRDS 2013 annual data report: atlas of chronic kidney disease and end-stage renal disease. Bethesda: National Institutes of Health, National Institute of Diabetes and Digestive and Kidney Diseases; 2013. Available from: https://www.usrds.org/atlas13.aspx.

[2] Kim YL. Update on mechanisms of ultrafiltration failure. Perit Dial Int 2009;29 Suppl 2:S123-S127.

[3] Yáñez-Mó M, Lara-Pezzi E, Selgas R, et al. Peritoneal dialysis and epithelial-to-mesenchymal transition of mesothelial cells. N Engl J Med 2003;348:403-413.

[4] Do JY, Kim YL, Park JW, et al. The effect of low glucose degradation product dialysis solution on epithelial-to-mesenchymal transition in continuous ambulatory peritoneal dialysis patients. Perit Dial Int 2005;25 Suppl 3:S22-S25.

[5] Oh EJ, Ryu HM, Choi SY, et al. Impact of low glucose deg- 
radation product bicarbonate/lactate-buffered dialysis solution on the epithelial-mesenchymal transition of peritoneum. Am J Nephrol 2010;31:58-67.

[6] Fernández-Perpén A, Pérez-Lozano ML, Bajo MA, et al. Influence of bicarbonate/low-GDP peritoneal dialysis fluid (BicaVera) on in vitro and ex vivo epithelial-to-mesenchymal transition of mesothelial cells. Perit Dial Int 2012;32: 292-304.

[7] Cho Y, Johnson DW, Craig JC, Strippoli GF, Badve SV, Wiggins KJ. Biocompatible dialysis fluids for peritoneal dialysis. Cochrane Database Syst Rev 2014;(3):CD007554.

[8] Azuma H, Banno K, Yoshimura T. Pharmacological properties of N-(3',4'-dimethoxycinnamoyl) anthranilic acid (N-5'), a new anti-atopic agent. Br J Pharmacol 1976;58:483-488.

[9] Suzawa H, Kikuchi S, Arai N, Koda A. The mechanism involved in the inhibitory action of tranilast on collagen biosynthesis of keloid fibroblasts. Jpn J Pharmacol 1992;60:9196.

[10] Taniguchi S, Yorifuji T, Hamada T. Treatment of linear localized scleroderma with the anti-allergic drug, tranilast. Clin Exp Dermatol 1994;19:391-393.

[11] Darakhshan S, Pour AB. Tranilast: a review of its therapeutic applications. Pharmacol Res 2015;91:15-28.

[12] Kaneyama T, Kobayashi S, Aoyagi D, Ehara T. Tranilast modulates fibrosis, epithelial-mesenchymal transition and peritubular capillary injury in unilateral ureteral obstruction rats. Pathology 2010;42:564-573.

[13] Ohshio Y, Teramoto K, Hashimoto M, Kitamura S, Hanaoka J, Kontani K. Inhibition of transforming growth factor- $\beta$ release from tumor cells reduces their motility associated with epithelial-mesenchymal transition. Oncol Rep 2013; 30:1000-1006.

[14] Li SS, Liu QF, He AL, Wu FR. Tranilast attenuates TGF- $\beta 1$ induced epithelial-mesenchymal transition in the NRK-52E cells. PakJ Pharm Sci 2014;27:51-55.

[15] Saito H, Fushida S, Harada S, et al. Importance of human peritoneal mesothelial cells in the progression, fibrosis, and control of gastric cancer: inhibition of growth and fibrosis by tranilast. Gastric Cancer 2018;21:55-67.

[16] Kaneko K, Hamada C, Tomino Y. Peritoneal fibrosis intervention. Perit Dial Int 2007;27 Suppl 2:S82-S86.

[17] Kang SH, Kim SO, Cho KH, Park JW, Yoon KW, Do JY. Paricalcitol ameliorates epithelial-to-mesenchymal transition in the peritoneal mesothelium. Nephron Exp Nephrol 2014;126:1-7.

[18] Park SH, Lee EG, Kim IS, Kim YJ, Cho DK, Kim YL. Effect of glucose degradation products on the peritoneal membrane in a chronic inflammatory infusion model of peritoneal dialysis in the rat. Perit Dial Int 2004;24:115-122.

[19] De Vriese AS, Tilton RG, Mortier S, Lameire NH. Myofibroblast transdifferentiation of mesothelial cells is mediated by RAGE and contributes to peritoneal fibrosis in uraemia. Nephrol Dial Transplant 2006;21:2549-2555.

[20] Lan HY. Smads as therapeutic targets for chronic kidney disease. Kidney Res Clin Pract 2012;31:4-11.

[21] Derynck R, Zhang YE. Smad-dependent and Smad-independent pathways in TGF-beta family signalling. Nature 2003;425:577-584.

[22] Zhang YE. Non-Smad pathways in TGF-beta signaling. Cell Res 2009;19:128-139.

[23] Wang H, Zhang G, Zhang H, et al. Acquisition of epithelialmesenchymal transition phenotype and cancer stem celllike properties in cisplatin-resistant lung cancer cells through AKT/ $\beta$-catenin/Snail signaling pathway. Eur J Pharmacol 2014;723:156-166.

[24] Tomino Y. Mechanisms and interventions in peritoneal fibrosis. Clin Exp Nephrol 2012;16:109-114.

[25] Ji S, Deng H, Jin W, et al. Beta-catenin participates in dialysate-induced peritoneal fibrosis via enhanced peritoneal cell epithelial-to-mesenchymal transition. FEBS Open Bio 2017;7:265-273.

[26] Yan P, Tang H, Chen X, et al. Tamoxifen attenuates dialysate-induced peritoneal fibrosis by inhibiting GSK-3 $\beta$ / $\beta$-catenin axis activation. Biosci Rep 2018;38:BSR20180240.

[27] Kazama I, Baba A, Endo Y, et al. Mast cell involvement in the progression of peritoneal fibrosis in rats with chronic renal failure. Nephrology (Carlton) 2015;20:609-616.

[28] Chung AC, Lan HY. MicroRNAs in renal fibrosis. Front Physiol 2015;6:50.

[29] Qin W, Chung AC, Huang XR, et al. TGF- $\beta /$ Smad3 signaling promotes renal fibrosis by inhibiting miR-29. J Am Soc Nephrol 2011;22:1462-1474.

[30] Chuang TD, Khorram O. Tranilast inhibits genes functionally involved in cell proliferation, fibrosis, and epigenetic regulation and epigenetically induces miR-29c expression in leiomyoma cells. Reprod Sci 2017;24:1253-1263. 